\title{
Cardiac shock wave therapy protects cardiomyocytes from hypoxia-induced injury by modulating miR-210
}

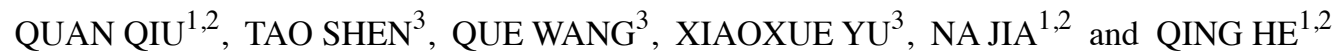 \\ ${ }^{1}$ Graduate School of Peking Union Medical College and Chinese Academy of Medical Sciences; ${ }^{2}$ Department of Cardiology; \\ ${ }^{3}$ The MOH Key Laboratory of Geriatrics, National Center of Gerontology, Beijing Hospital, Beijing 100730, P.R. China
}

Received May 18, 2019; Accepted October 17, 2019

DOI: $10.3892 / \mathrm{mmr} .2019 .10892$

\begin{abstract}
Cardiac shock wave therapy (SWT) has been described as a novel therapeutic strategy that is able to alleviate myocardial ischemic injury. microRNA (miRNA/miR)-210 plays a cytoprotective role in cardiomyocytes in response to hypoxia by regulating cell apoptosis. The aim of the present study was to investigate whether cardiac SWT could protect cardiomyocytes from hypoxia-induced injury by regulating miR-210 expression. The murine adult cardiomyocyte cell line HL-1 was incubated for $5 \mathrm{~h}$ in hypoxic conditions, followed by reoxygenation for $12 \mathrm{~h}$ and treatment with SWT immediately following hypoxia in the present study. The cell viability was determined using an MTS assay. Western blot analyses were performed in order to detect cell signaling changes. Reactive oxygen species production was detected using dihydroethidium staining, and malondialdehyde levels were measured using the thiobarbituric acid method. miRNA and mRNA expression levels were confirmed via reverse transcription-quantitative PCR. Apoptosis was evaluated by means of flow cytometry. HL-1 cells were then transfected with miR-210 mimics or inhibitors in order to alter miR-210 expression levels, and the effects on HL-1 cells were determined. Hypoxia led to elevated oxidative stress, enhanced cell apoptosis and upregulated miR-210 expression levels in HL-1 cells, while SWT could alleviate hypoxia-induced cell injury and further promote miR-210 expression. miR-210 overexpression decreased apoptosis and oxidative stress during hypoxic stress in HL-1 cells, whereas inhibition of miR-210 increased cell apoptosis and promoted oxidative stress. Furthermore, miR-210 inhibition could reverse the effects of SWT on HL-1 cells. Finally, the mRNA analysis revealed that SWT significantly attenuated apoptosis-inducing factor mitochondrion-associated 3 and
\end{abstract}

Correspondence to: Professor Qing He, Graduate School of Peking Union Medical College and Chinese Academy of Medical Sciences, Beijing Hospital, 1 Dahua Road, Dongdan, Beijing 100730, P.R. China

E-mail: heqingli2001@yahoo.com

Key words: microRNA-210, hypoxia, shock wave therapy, apoptosis, oxidative stress caspase 8 associated protein 2 mRNA expression levels in cardiomyocytes exposed to hypoxia, which were two targets of miR-210. SWT could exert cardioprotective effects against hypoxia-induced cardiac injury by modulating miR-210.

\section{Introduction}

Timely and successful myocardial reperfusion with either primary percutaneous coronary intervention or thrombolytic therapy has resulted in a significant decrease in infarct area size and mortality rates of acute myocardial infarction (AMI) (1). However, ischemic heart disease (IHD) following AMI is one of the principal causes of death and disability worldwide (2). MicroRNAs (miRNAs/miRs) are a class of small non-coding RNAs $\sim 20$ ribonucleotides in length that negatively regulate target genes by binding to sequences in the 3'-untranslated regions of target messenger RNAs (3). Currently, a growing body of evidence has indicated that miRNAs may play an important role in the processes that lead to the pathophysiological consequences of IHD by altering key signaling elements and regulating proliferation, differentiation and survival in mRNA levels (4-7).

miR-210 has been demonstrated to be markedly upregulated in response to hypoxia in a broad spectrum of cell types (8), including cardiomyocytes. A murine model study revealed that miR-210 overexpression in the heart using minicircle vectors enhanced cardiac function, decreased infarct size, inhibited apoptosis and induced neovascularization following AMI (9). Other studies have revealed that apoptosis-inducing factor mitochondrion-associated 3 (AIFM3) and caspase 8 associated protein 2 (Casp8ap2), known as proapoptotic proteins, were inhibited by miR-210 overexpression $(9,10)$. miR-210 has also been demonstrated as being involved in several other cellular functions, from stem cell survival $(9,11)$ and reactive oxygen species (ROS) generation (10) to angiogenesis (12). Thus, miR-210 is suggested to be a potential therapeutic target for treating IHD.

Cardiac shock wave therapy (SWT) is a promising, noninvasive and safe modality that has been demonstrated to alleviate myocardial ischemia and improve cardiac function in patients with IHD $(13,14)$. The improvement of myocardial perfusion and exercise capacity has been observed in numerous clinical trials $(15,16)$. In vitro studies have indicated that cardiac SWT decreased hypoxia-induced apoptosis in H9c2 cells by 
activating the PI3K-Akt pathway (17). A recent report revealed that cardiac SWT protected cardiomyocytes from apoptosis by attenuating cytochrome $\mathrm{c}$ release from the mitochondria in an in vivo rat AMI model (18). However, few studies have focused on miRNAs in regard to their protective effects during cardiac SWT. Taken together, an evaluation of the influence of cardiac SWT on miR-210 following myocardial ischemic injury would be of use.

The present study used an in vitro model of AMI in order to investigate whether cardiac SWT could protect cardiomyocytes against hypoxia through modulating miR-210 and the underlying molecular mechanisms.

\section{Materials and methods}

Reagents. Dulbecco's Modified Eagle's medium (DMEM), RPMI-1640 medium and protease inhibitor cocktails were purchased from Sigma-Aldrich; Merck KGaA. Trypsin-EDTA, PBS, penicillin/streptomycin and fetal bovine serum (FBS) were from Thermo Fisher Scientific, Inc. Antibodies (Abs) directed against GAPDH, Bcl-2, Bax, p38 mitogen-activated protein kinase (MAPK), phosphorylated (p)-p38MAPK, Akt, p-Akt, horseradish peroxidase (HRP)-coupled anti-rabbit IgG secondary $\mathrm{Ab}$ and lysis buffer were purchased from Cell Signaling Technology, Inc. Protein concentration was determined by bicinchoninic acid (BCA) protein assay kit from Pierce; Thermo Fisher Scientific, Inc. Immobilon Western HRP Substrate was purchased from Merck KGaA. Fluorescent assays for apoptosis was from Beijing Solarbio Science \& Technology Co., Ltd. The Cell Titer $96^{\circledR}$ AQueous One Solution Cell Proliferation Assay was obtained from Promega Corporation. miR-210 mimics, miR-210 inhibitors and negative controls (NC) of miRNA were all designed and synthesized by Sangon Biotech Co., Ltd. The sequences of miR-210 inhibitor negative controls and mimics negative controls were as follows (5' to $\left.3^{\prime}\right)$ : miR-210 inhibitor negative controls, CAGUACUUU UGUGUAGUACAA; miR-210 mimics negative controls sense, UUCUCCGAACGUGUCACGUTT; and miR-210 mimics negative controls antisense, ACGUGACACGUUCGG AGAATT. TRIzol ${ }^{\circledR}$ and Lipofectamine ${ }^{\circledR}$ RNAiMAX reagent were obtained from Thermo Fisher Scientific, Inc. MicroRNA reverse transcription kit was from New England BioLabs, Inc. SYBR Green PCR Master Mix was purchased from Takara Biotechnology Co., Ltd. A lipid peroxidation malondialdehyde (MDA) assay kit was purchased from Beyotime Institute of Biotechnology (cat. no. S0131).

HL-1 cell culture. HL-1 cells were provided by Dr William Claycomb (Louisiana State University Health Science Center), an immortalized cell line derived from mouse atrial cardiac myocytes, were cultured in DMEM supplemented with $10 \%$ FBS, $100 \mathrm{U} / \mathrm{ml}$ penicillin and $100 \mu \mathrm{g} / \mathrm{ml}$ streptomycin. Cells were maintained at $37^{\circ} \mathrm{C}$ in a humidified chamber with an atmosphere of $95 \%$ air and $5 \% \mathrm{CO}_{2}$.

Hypoxia treatment. When the cells reached a confluence of $60-70 \%$, HL-1 cells were cultured in FBS-free media for $24 \mathrm{~h}$ before all experiments. To mimic ischemic injury in vivo, cells were exposed to ischemia-mimetic solution as previously described (19). Subsequently, the cells were incubated in a 3-gas hypoxic chamber (HERAcell VIOS 160i, Thermo Fisher Scientific, Inc.) equilibrated with $94 \% \mathrm{~N}_{2}, 5 \% \mathrm{CO}_{2}$ and $1 \% \mathrm{O}_{2}$ for $5 \mathrm{~h}$.

SWT. After $5 \mathrm{~h}$ of hypoxia, cells were subjected to SWT as previously described (20). In brief, the HL-1 cells were digested with trypsin after hypoxia, transferred to centrifuge tubes $\left(1 \mathrm{ml}, 5 \times 10^{6}\right.$ cells $\left./ \mathrm{ml}\right)$ and treated with a shock wave device (Duolith VET; Storz Medical AG). Both the ultrasonic probe and the tubes were submerged in a water bath at $37^{\circ} \mathrm{C}$. Then, 200 pulses of shock waves with an energy of $0.05 \mathrm{~mJ} / \mathrm{mm}^{2}$ were administered to the cells. After SWT, cells were placed in standard medium under normoxic conditions for $12 \mathrm{~h}$ before harvest.

MTS assay. Cell viability was measured by Cell Titer 96 AQueous One Solution Cell Proliferation Assay. In brief, HL-1 cells were obtained after hypoxia and SWT treatment and re-suspended to a density of $5 \times 10^{6}$ cells $/ \mathrm{ml}$. Then, cells were seeded in 24-well plates in quadruplicate. MTS reagent (45 $\mu \mathrm{l}$ ) was added to each well of the 24-well plates with $225 \mu \mathrm{l}$ of culture medium. After $4 \mathrm{~h}$, the absorbance was detected at $490 \mathrm{~nm}$ using a microplate reader.

Flow cytometry. Cell apoptosis was analyzed using flow cytometry after fluorescein isothiocyanate-conjugated Annexin V (FITC-Annexin V; Beijing Solarbio Science \& Technology Co., Ltd.) and propidium iodide (PI) staining. The staining procedure was performed according to the instructions. HL-1 cells in early logarithmic growth phase were seeded in 6-well plates, harvested by centrifugation at $800 \mathrm{~g}$ for $5 \mathrm{~min}$ at room temperature, and then washed twice with PBS. Cells were resuspended in $100 \mu 1$ of $1 \mathrm{X}$ binding buffer to a density of $1 \times 10^{6}$ cells $/ \mathrm{ml}, 5 \mu \mathrm{l}$ of Annexin V-FITC was added, and the cells were incubated in the dark at room temperature for $10 \mathrm{~min}$. Then, $5 \mu \mathrm{l}$ of PI was added and the cells were incubated in the dark at room temperature for another $5 \mathrm{~min}$. Finally, $500 \mu 1$ of $1 \mathrm{X}$ binding buffer was added to the mixture, which was then loaded onto a flow cytometer (FACSCalibur; BD Biosciences) for the apoptosis analysis using FlowJo 7.6.1 (BD Biosciences). Cells that were considered viable were both Annexin V and PI negative, while cells that were in early apoptosis were Annexin $\mathrm{V}$ positive and PI negative, and necrotic cells were both Annexin V and PI positive.

Lipid peroxidation. MDA is a natural byproduct of lipid peroxidation and used as a reliable marker to measure the level of oxidative stress. Free MDA reacts with thiobarbituric acid (TBA) and generates an MDA-TBA adduct, which can be quantified colorimetrically (optical density $532 \mathrm{~nm}$ ). MDA levels were measured using the TBA method according to the manufacturer's protocol.

Measuring intracellular ROS levels. ROS generation was measured with dihydroethidium (DHE; Invitrogen; Thermo Fisher Scientific, Inc.) staining and observed under a fluorescent microscope (Olympus BX51; Olympus Corporation; magnification, x50). Briefly, When the HL-1 cells reached the appropriate confluence (70-80\%) in 24-well plates, the cells were incubated with DHE $(8 \mu \mathrm{m})$ for $30 \mathrm{~min}$ at $37^{\circ} \mathrm{C}$ in the 
dark, and then washed with PBS. Following the manufacturer's protocols, DHE was alternately excited at a wavelength of $535 \mathrm{~nm}$ and emitted at a wavelength of $512 \mathrm{~nm}$. The fluorescence was measured and expressed as a percentage of the integrated optical density in the control.

Western blot analysis. HL-1 cells were lysed in ice-cold lysis buffer (Cell Signaling Technology, Inc.) supplemented with phosphatase and protease inhibitors. Cell lysates were incubated on ice for $5 \mathrm{~min}$ and centrifuged at 13,000 $\mathrm{g}$ for $15 \mathrm{~min}$ $\left(4^{\circ} \mathrm{C}\right)$. Protein concentrations of the supernatants were determined using a Pierce ${ }^{\mathrm{TM}}$ BCA Protein Assay Kit. All samples were boiled for $10 \mathrm{~min}$ at $100^{\circ} \mathrm{C}$. Soluble proteins $(20 \mu \mathrm{g} / \mathrm{lane})$ were electrophoresed on $12 \%$ SDS polyacrylamide gels and transferred to polyvinylidene fluoride membranes that were then blocked with TBS with $0.1 \%$ Tween-20 buffer containing $5 \%$ non-fat milk for $2 \mathrm{~h}$ at room temperature. Membranes were incubated overnight at $4^{\circ} \mathrm{C}$ with specific primary Abs $(1: 1,000)$ against GAPDH (cat. no. 5174), Bcl-2 (cat. no. 2772), p38 MAPK (cat. no. 8690), p-p38 MAPK (cat. no. 4511), Akt (cat. no. 4691) and p-Akt (cat. no. 4060), and then incubated with secondary $\mathrm{Ab}(1: 5,000$; ct. no. 7074) for $2 \mathrm{~h}$ at room temperature. The immunoreaction was visualized with Immobilon Western HRP substrate. Densitometry was performed using ImageJ software Java 1.8.0 (National Institutes of Health).

Reverse transcription-quantitative PCR (RT-qPCR). Total RNAs from cardiomyocytes were isolated by TRIzol ${ }^{\circledR}$ reagent. The concentration of RNA was detected using a NanoDrop ${ }^{\mathrm{TM}}$ 2,000 Spectrophotometer (NanoDrop Technologies; Thermo Fisher Scientific, Inc.). Using a microRNA reverse transcription kit (New England BioLabs, Inc.), 800 ng of total RNA was reverse transcribed. The RNA specific primers and SYBR Green were used for RT-qPCR to examine the relative quantification of RNAs with a Bio-Rad iCycler RT-PCR Detection System (Bio-Rad Laboratories, Inc.). The thermocycling conditions were: $95^{\circ} \mathrm{C}$ for $10 \mathrm{~min}, 95^{\circ} \mathrm{C}$ for $15 \mathrm{sec}$ and $60^{\circ} \mathrm{C}$ for $60 \mathrm{sec}$. Then, steps 2-3 were repeated for 40 cycles. U6 and GAPDH were chosen as internal controls. Each reaction was performed in triplicate, and analysis was performed by the $2^{-\Delta \Delta \mathrm{Cq}}$ method (21). The primers are shown in Table I.

Transient transfection. HL-1 cells (70\% confluent) were transfected with miR-210 mimics $(20 \mu \mathrm{M})$ or inhibitors $(20 \mu \mathrm{M})$ or miRNA negative controls using Lipofectamine-RNAiMAX reagent according to the manufacturer's protocols. After $24 \mathrm{~h}$ of transfection, cells were treated with hypoxia and SWT.

Monodansylcadaverine (MDC) staining. The MDC kit was obtained from Nanjing KeyGen Biotech Co., Ltd. The cells were washed twice with $300 \mu 1 \mathrm{lX}$ washing buffer. Then, $100 \mu \mathrm{l}$ working solution was added to each well. The cells were incubated at room temperature for $20 \mathrm{~min}$ in the dark. The cells were washed four times with $1 \mathrm{X}$ washing buffer to remove redundant dye. Finally, cells were visualized using a fluorescent microscope (magnification, $x 50$ ) with an excitation wavelength of $355 \mathrm{~nm}$ and an emission filter of $512 \mathrm{~nm}$.

Statistical analysis. Data are presented as the mean \pm SEM. Student's t-test and one-way ANOVA with Bonferroni
Table I. Primers for RT-qPCR.

\begin{tabular}{|c|c|}
\hline Gene & Primer sequence $\left(5^{\prime}-3^{\prime}\right)$ \\
\hline \multirow[t]{4}{*}{ U6 } & Forward: GCGCGTCGTGAAGCGTTC \\
\hline & Universal: GTGCAGGGTCCGAGGT \\
\hline & Stem loop: GTCGTATCCAGTGCAGGGTCCG \\
\hline & AGGTATTCGCACTGGATACGACAAAATATG \\
\hline \multirow[t]{4}{*}{ miR-210 } & Forward: GTGCGTGTGACAGCGGC \\
\hline & Universal: GTGCAGGGTCCGAGGT \\
\hline & Stem loop: GTCGTATCCAGTGCAGGGTCCG \\
\hline & AGGTATTCGCACTGGATACGACTCAGCC \\
\hline \multirow[t]{2}{*}{ GAPDH } & Forward: TGGCCTTCCGTGTTCCTACC \\
\hline & Reverse: TGTAGGCCATGAGGTCCACCAC \\
\hline \multirow[t]{2}{*}{ Casp8ap2 } & Forward: ACAGTGAAGCAAGGACGGAG \\
\hline & Reverse: TCGAGTGTGTGGCCTTTCTC \\
\hline \multirow[t]{2}{*}{ AIFM3 } & Forward: CTGGCCTAGTGTGTGCAGAA \\
\hline & Reverse: CCGGTCATAGGGCAGATGTC \\
\hline
\end{tabular}

RT-qPCR, reverse transcription-quantitative PCR; miR-210, microRNA-210; Casp8ap2, caspase 8 associated protein 2; AIFM3, apoptosis-inducing factor mitochondrion-associated 3 .

correction were performed to compare two conditions and multiple groups, respectively. All statistical calculations were performed by GraphPad Prism 5 software (GraphPad Software, Inc.) and $\mathrm{P}<0.05$ was considered to indicate a statistically significant difference.

\section{Results}

Hypoxia induces cell injury in HL-1 cardiomyocytes. HL-1 cardiomyocytes have previously been applied in hypoxia-induced studies, and have been demonstrated as a cell line for cardiomyocyte study (22). Therefore, the present study created an in vitro model of myocardial ischemia using HL-1 cells. When using the MTS assay, cell viability was significantly decreased by $29.6 \pm 1.6 \%$ after $5 \mathrm{~h}$ of exposure to hypoxia, followed by $12 \mathrm{~h}$ of reoxygenation when compared with the control, which was considered to be a moderate injury (Fig. 1A). In order to further investigate hypoxia-induced injury in cardiomyocytes, an Annexin V/PI staining assay was used to detect cardiomyocyte apoptosis. Hypoxia significantly increased the apoptotic rate compared with normoxic cells (Fig. 1B). Furthermore, western blot analysis verified that hypoxia induced a decrease in the $\mathrm{Bcl}-2 / \mathrm{Bax}$ ratio, indicating an increase in cell apoptosis (Fig. 2).

SWT alleviates hypoxia-induced oxidative stress and apoptosis in HL-1 cells. In order to investigate the effect of SWT on hypoxic injury, SWT was applied to HL-1 cells during the first $10 \mathrm{~min}$ of reperfusion. SWT led to increased cell viability (Fig. 1A), a significant decrease in apoptosis as manifested by reduced apoptotic cells (Fig. 1B) and an elevated Bcl-2/Bax ratio specifically in hypoxic cells (Fig. 2), which was demonstrated by the morphological changes of HL1 cells and the decreased number of dead cells after 

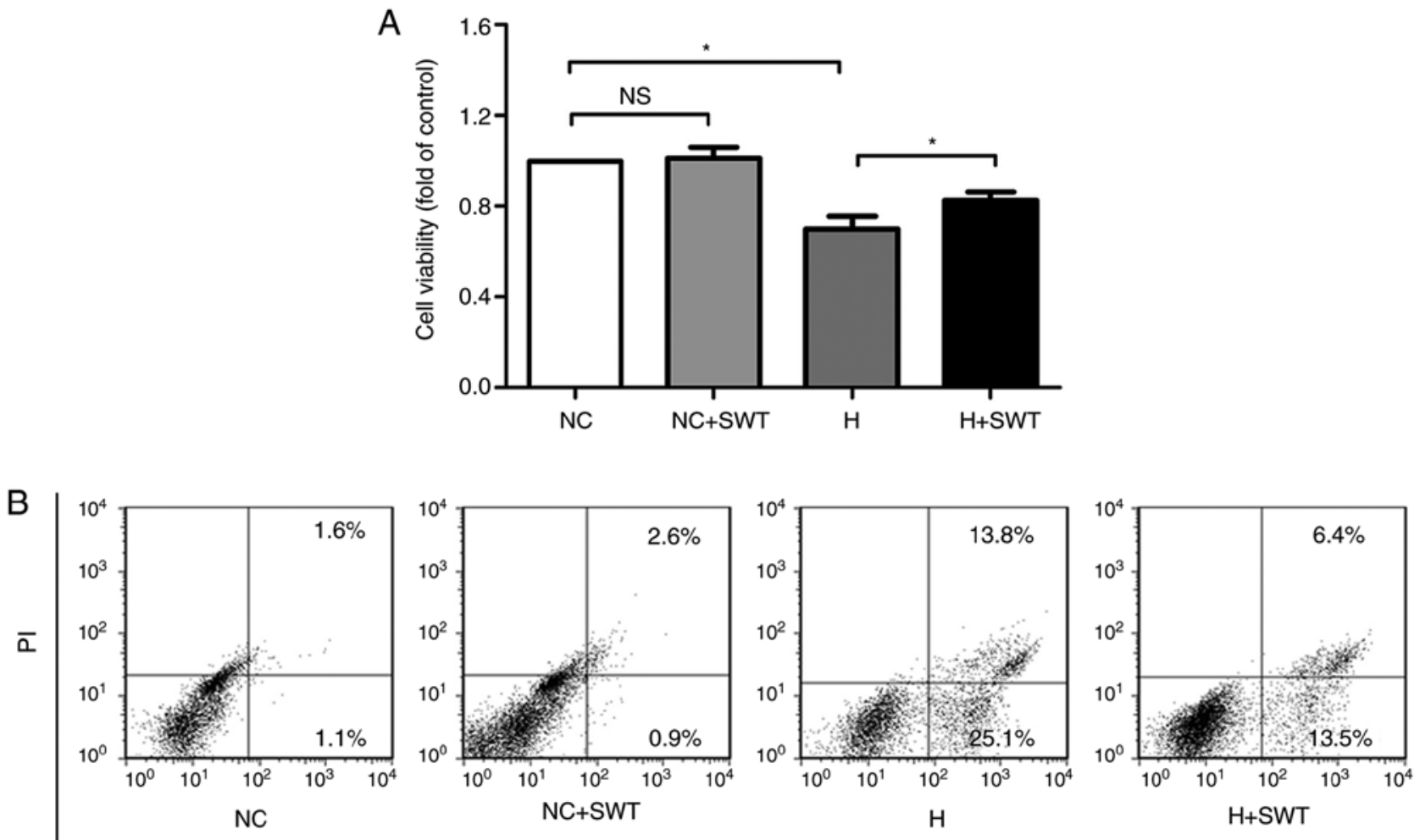

Annexin V/FITC
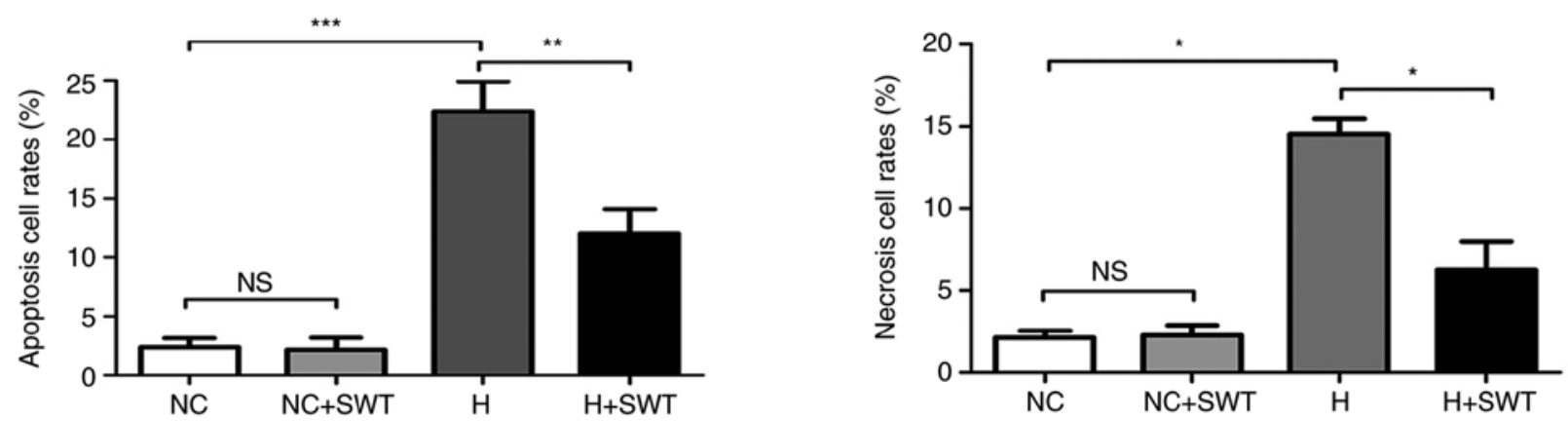

Figure 1. Hypoxia induces cell injury in HL-1 cells, which can be reversed by SWT. (A) Cell viability was determined by MTS assay (n=4). (B) Annexin V/PI double staining was used to assess the apoptotic rates of HL-1 cells in the NC, NC + SWT, hypoxia and hypoxia + SWT groups $(n=4)$. ${ }^{*} \mathrm{P}<0.05,{ }^{* *} \mathrm{P}<0.01$, ${ }^{* * * *} \mathrm{P}<0.001$. SWT, shock wave therapy; ns, not significant; NC, negative control; H, hypoxia; PI, propidium iodide.

SWT (Fig. S1). Accumulating evidence has suggested that excessive ROS generation during ischemia could stimulate apoptosis (23). Therefore, the present study determined whether SWT inhibited ROS production in the hypoxic model. Using DHE staining, the present study revealed increased ROS production following hypoxia treatment, and ROS levels were decreased by SWT (Fig. 3A). Excessive production of ROS results in lipid peroxidation that generates oxidized by-products, such as MDA (24). Thus, the present study detected MDA levels using the TBA method. As presented in Fig. 3B, the hypoxic challenge caused a markedly increased MDA level, which was downregulated by SWT (NC group vs. HR group vs. HR + SWT group, $1.0780 \pm 0.1229 \mathrm{nmol} / \mathrm{mg}$ protein vs. $3.5000 \pm 0.1600 \mathrm{nmol} / \mathrm{mg}$ protein vs. $2.8000 \pm 0.2300 \mathrm{nmol} / \mathrm{mg}$ protein, respectively). Furthermore, in order to determine the molecular mechanisms underlying the protective effects of SWT on hypoxic injury, cell survival-associated proteins, including p38MAPK and Akt, were measured. Western blot analysis revealed that cells exposed to hypoxia exhibited significantly decreased expression of p-Akt and significantly increased expression of p-p38MAPK (Fig. 2). In addition, it was revealed that SWT significantly suppressed hypoxia-induced p38MAPK phosphorylation and increased p-Akt in HL-1 cells. Overall, these data suggested that SWT could reverse oxidative stress and apoptosis in HL-1 cells exposed to hypoxia.

SWT increased miR-210 level in cardiomyocytes exposed to hypoxia. Previous reports have demonstrated that miR- 210 is one of several hypoxia-induced miRNAs that may play an important role in cell survival (25). In order to investigate whether hypoxia regulated miR-210 in HL-1 cells, the present study used RT-qPCR to determine miR-210 levels in HL-1 cells following hypoxia treatment. As presented in Fig. 4A, hypoxia led to a substantial increase in miR-210 expression. Notably, the administration of SWT to the hypoxic cells resulted in a significant increase in miR-210 expression in comparison with the hypoxia group. 

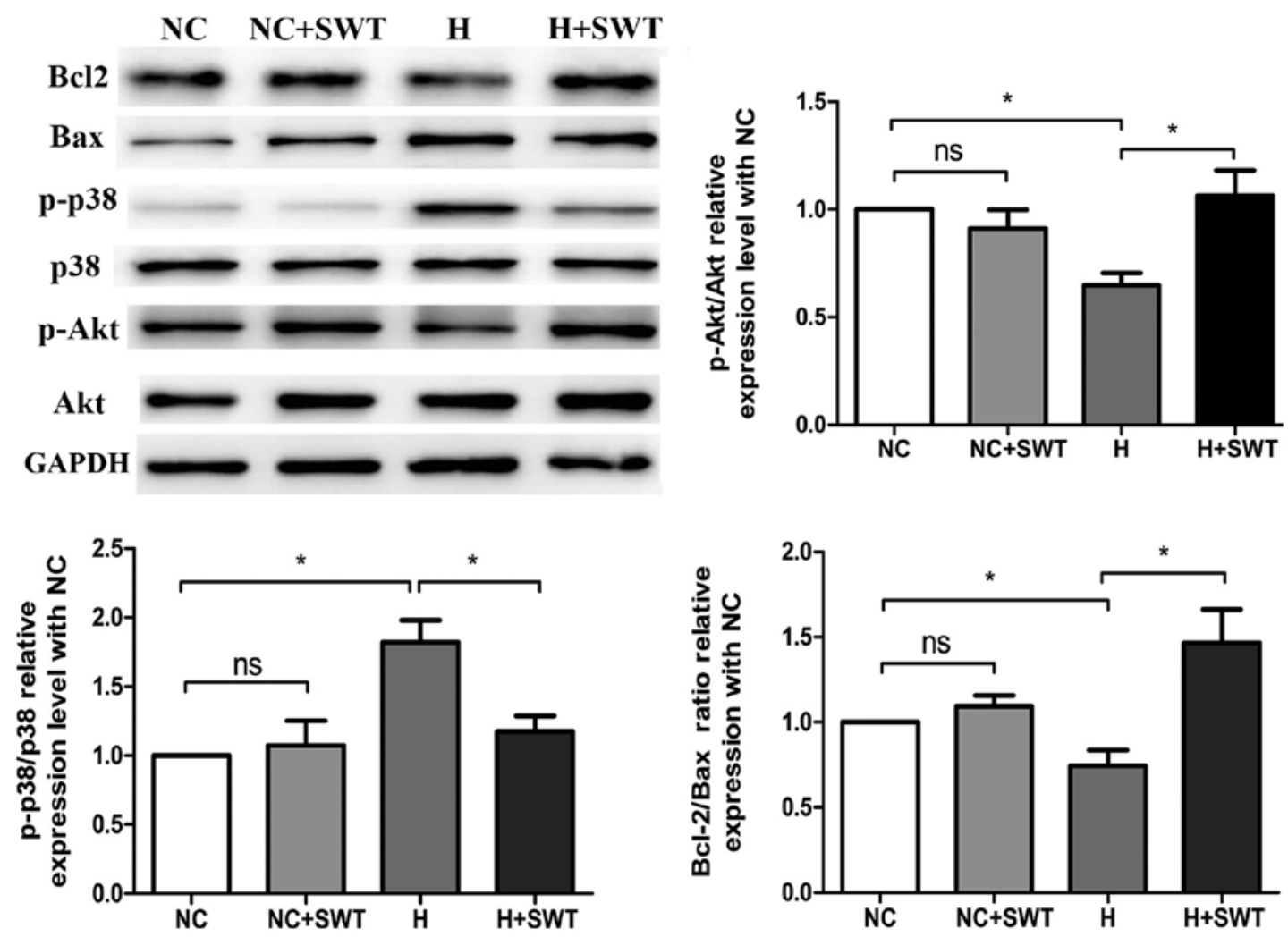

Figure 2. Western blotting and average data for Bcl-2, Bax, Akt, p-Akt, p38MAPK and p-p38MAPK levels in HL-1 cells exposed to hypoxia and SWT (n=4). ${ }^{*} \mathrm{P}<0.05$. MAPK, mitogen-activated protein kinase; SWT, shock wave therapy; ns, not significant; NC, negative control; H, hypoxia; MAPK, p, phosphorylated.
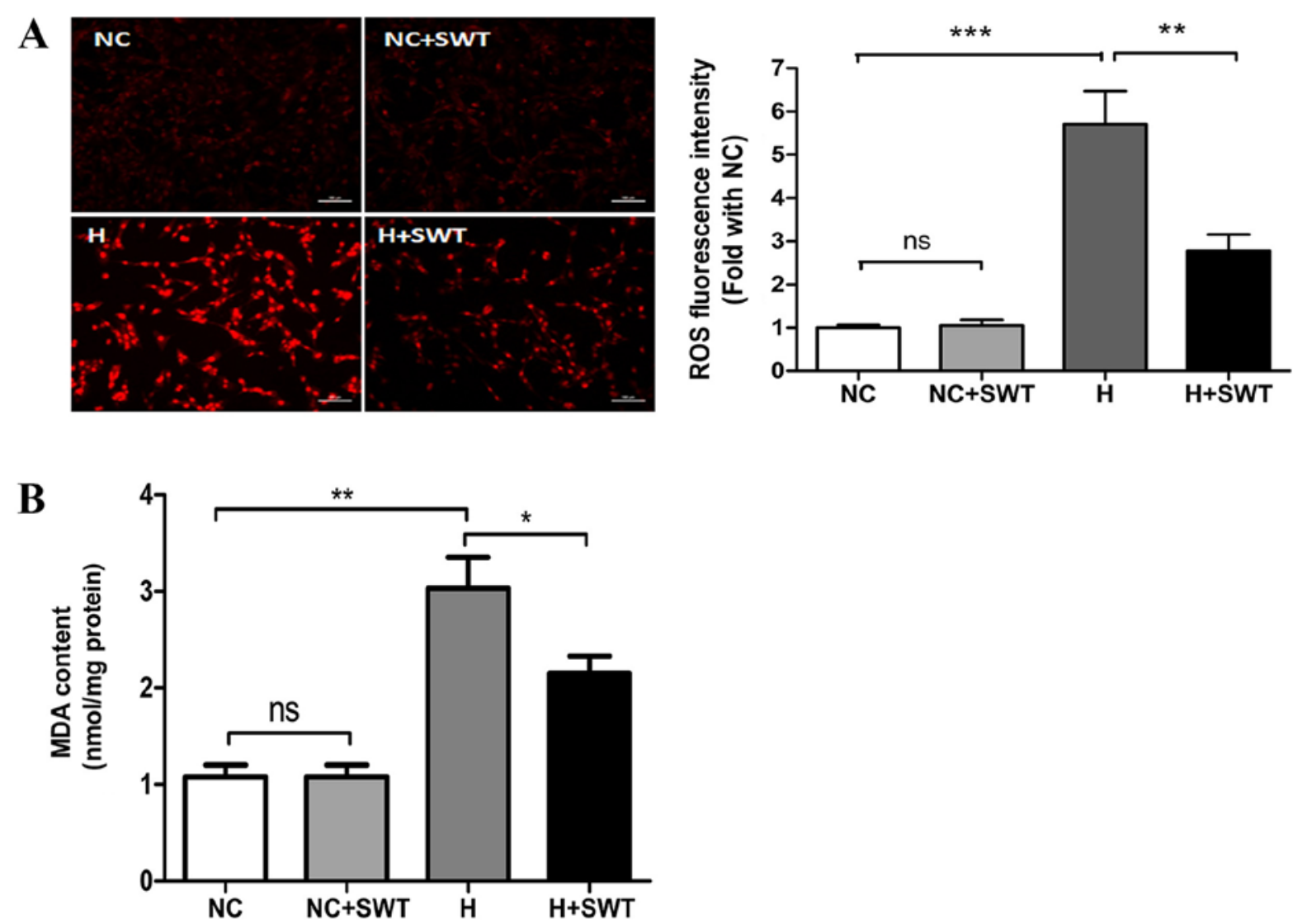

Figure 3. SWT alleviates hypoxia-induced oxidative stress in HL-1 cells. (A) ROS production, as measured using dihydroethidium staining, in the NC, NC + SWT, hypoxia and hypoxia + SWT groups (n=3; scale bar, $100 \mu \mathrm{m}$ ). (B) MDA levels were assessed using the thiobarbituric acid method in HL-1 cells exposed to hypoxia and SWT $(\mathrm{n}=4) .{ }^{*} \mathrm{P}<0.05,{ }^{* *} \mathrm{P}<0.01,{ }^{* * *} \mathrm{P}<0.001$. SWT, shock wave therapy; ROS, reactive oxygen species; NC, negative control; ns, not significant; $\mathrm{H}$, hypoxia; MDA, malondialdehyde. 
A

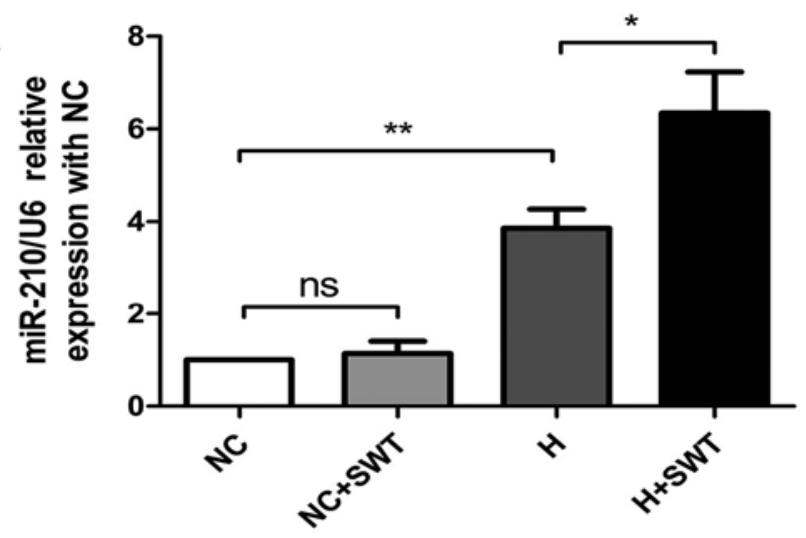

C

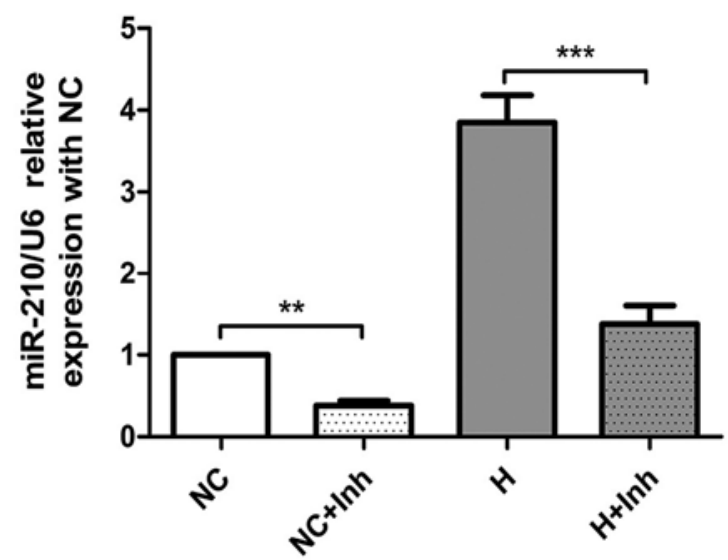

D

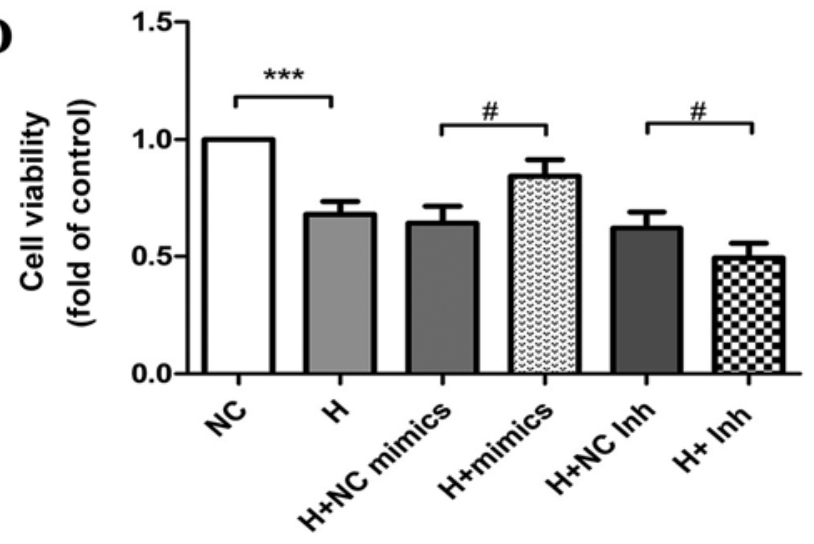

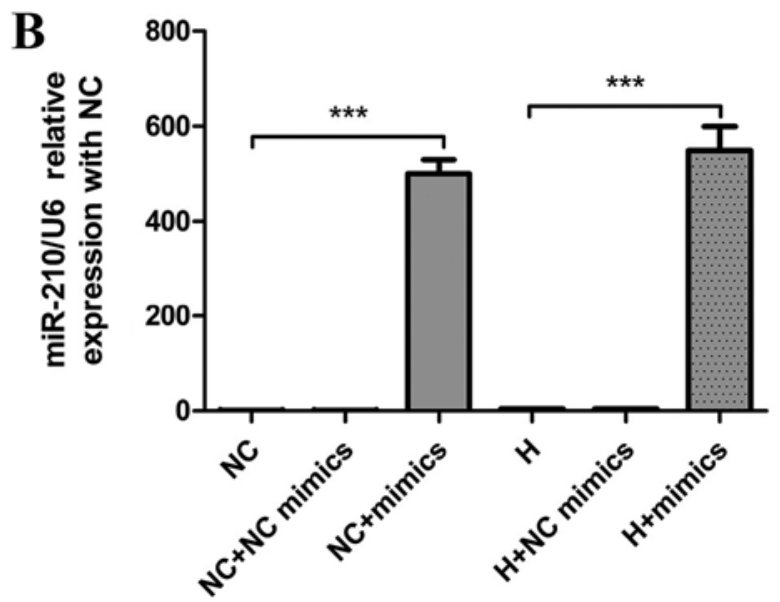

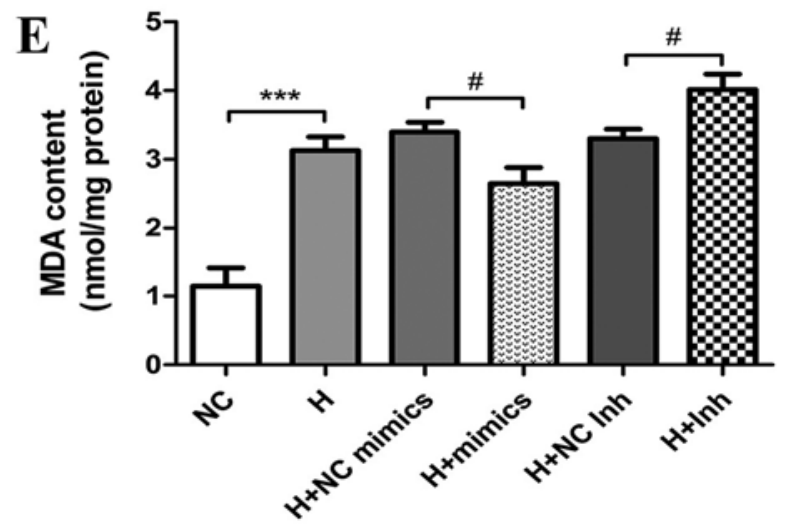

Figure 4. miR-210 protects cardiomyocytes against hypoxia and SWT enhances miR-210 expression in HL-1 cells exposed to hypoxia. (A) miR-210 expression in HL-1 cells exposed to hypoxia as demonstrated by RT-qPCR in the NC, NC + SWT, hypoxia, and hypoxia + SWT groups (n=4). (B) miR-210 expression in HL-1 cells exposed to normoxic and hypoxic conditions using RT-qPCR following the transfection of miR-210 mimics. Data are expressed as the relative fold change in comparison with the negative control $(\mathrm{n}=3)$. (C) miR-210 expression in HL-1 cells exposed to normoxic and hypoxic conditions as determined by RT-qPCR following the transfection of miR-210 inhibitors. Data are expressed as the relative fold change in comparison with the negative control (n=3). (D) Cell viability was assessed following the transfection of miR-210 mimics or miR-210 inhibitors into HL-1 cardiomyocytes compared with negative controls $(\mathrm{n}=4)$. (E) MDA levels were detected following the transfection of miR-210 mimics or miR-210 inhibitors into HL-1 cardiomyocytes $(\mathrm{n}=4)$. ${ }^{*} \mathrm{P}<0.05,{ }^{* *} \mathrm{P}<0.01$, ${ }^{* * * *} \mathrm{P}<0.001 ;{ }^{*} \mathrm{P}<0.05$. miR, microRNA; RT-qPCR, reverse transcription-quantitative PCR; NC, negative control; SWT, shock wave therapy; ns, not significant; $\mathrm{H}$, hypoxia; Inh, inhibitors; MDA, malondialdehyde.

miR-210 attenuates hypoxia-induced oxidative stress and apoptosis in cardiomyocytes. In order to investigate the effect of miR-210 on cardiomyocytes following hypoxia, miR-210 mimics and inhibitors were transfected in order to alter the expression of miR-210 in HL-1 cells. RT-qPCR analysis revealed that miR-210 expression in the mimics group was significantly increased compared with HL-1 cells transfected with the negative control in normoxic and hypoxic cells (Fig. 4B); in contrast, inhibitors transfection decreased miR-210 expression (Fig. 4C). Using an MTS assay, the data 

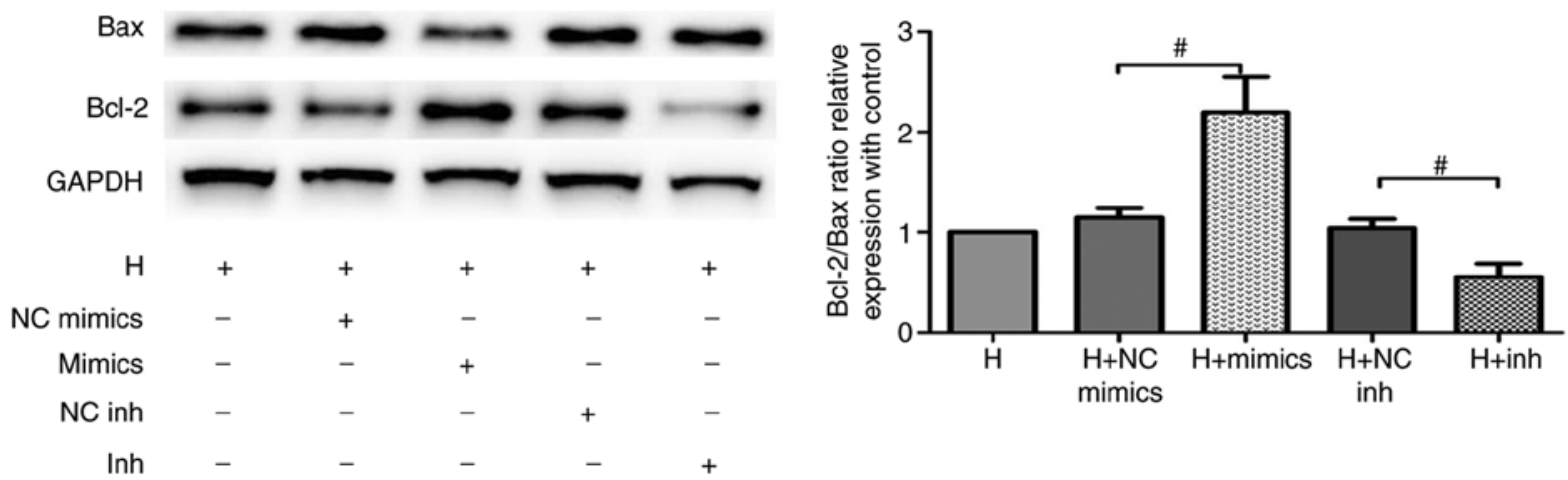

Figure 5. Western blotting of Bcl-2 and Bax. Levels of Bcl-2 and Bax in HL-1 cells exposed to hypoxia and transfected with miR-210 mimics or miR-210 inhibitors $(\mathrm{n}=3)$. " $\mathrm{P}<0.05$. miR, microRNA; ns, not significant; NC, negative control; H, hypoxia; Inh, inhibitors.
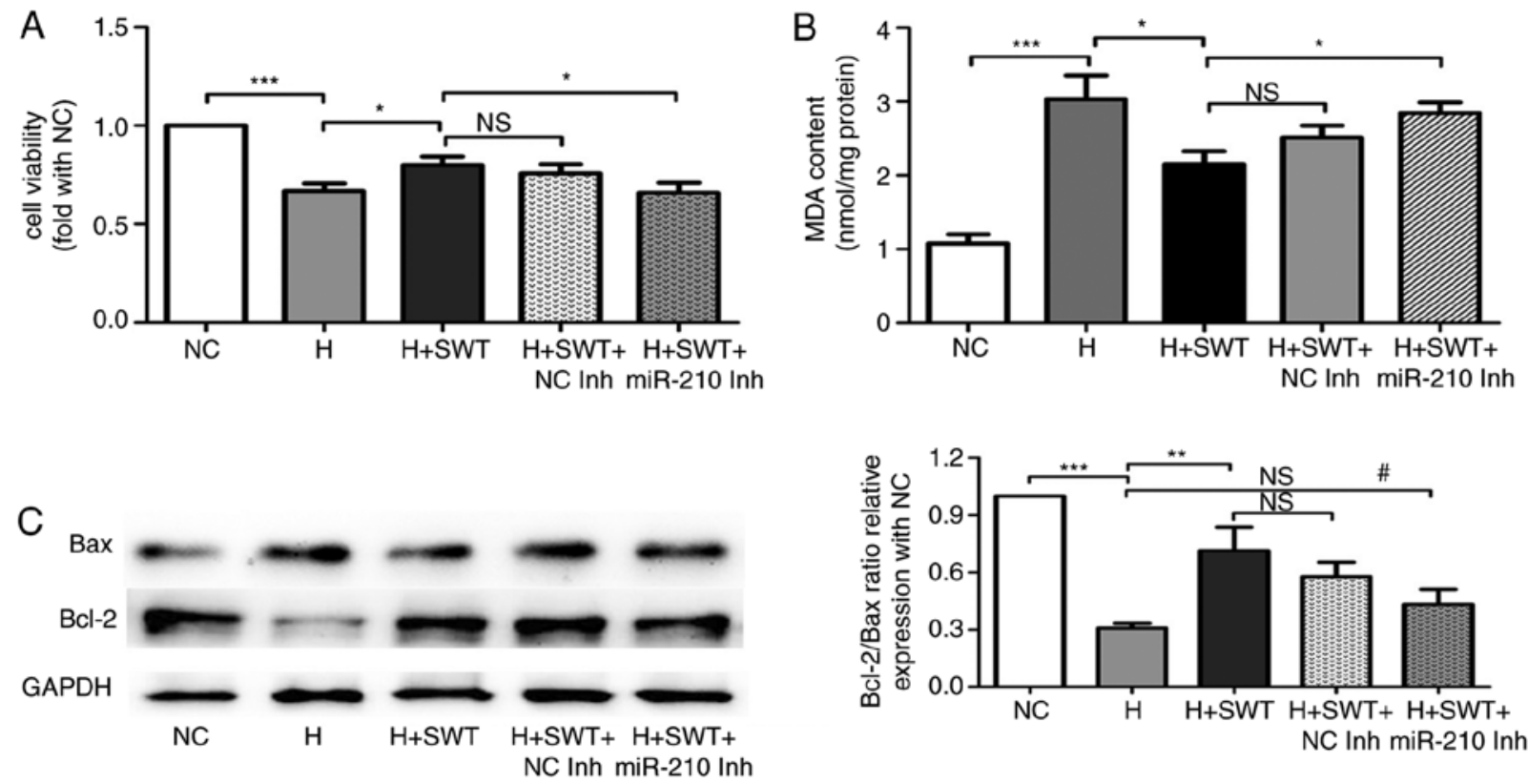

Figure 6. miR-210 inhibition blocks the protective effect of SWT. (A) Cell viability was determined using an MTS assay (n=4). (B) MDA levels were assessed using the thiobarbituric acid method $(\mathrm{n}=4)$. (C) Expression of apoptosis-associated proteins demonstrated by western blot analysis $(\mathrm{n}=3)$. ${ }^{*} \mathrm{P}<0.05,{ }^{* *} \mathrm{P}<0.01$, ${ }_{* * * *} \mathrm{P}<0.001 ;{ }^{*} \mathrm{P}<0.05$. miR, microRNA; SWT, shock wave therapy; ns, not significant; NC, negative control; H, hypoxia; Inh, inhibitors; MDA, malondialdehyde.

from the present study revealed that increased expression of miR-210 significantly increased cell viability, while inhibition of miR-210 decreased cell viability when compared with the respective controls (Fig. 4D). Consistently, oxidative stress was evaluated by MDA levels. As presented in Fig. 4E, overexpression of miR-210 led to a significant decrease in MDA level induced by hypoxia, while miR-210 inhibition resulted in the opposite effect. In order to determine whether miR-210 plays a role in regulating hypoxia-induced apoptosis in cardiomyocytes, Bax and Bcl-2 expression levels were evaluated via western blot analysis. As presented in Fig. 5, the Bcl-2/Bax ratio was significantly increased following transfection with miR-210 mimics when compared with controls, while miR-210 inhibitors decreased this level. Overall, these data demonstrated that miR-210 protected HL-1 cells against hypoxia-induced injury by inhibiting apoptosis and oxidative stress.

miR-210 inhibition blocks the protective effect of SWT. In order to verify whether SWT protects cardiomyocytes from hypoxia injury by regulating miR-210, HL-1 cells were transfected with miR-210 inhibitors or the negative controls prior to hypoxia treatment. The data in Fig. 6A and B demonstrated that the increased cell viability and decreased MDA levels regulated by SWT were reversed by miR-210 inhibition. Furthermore, the effects of SWT on apoptosis-associated proteins including Bax and Bcl-2 were attenuated by miR-210 inhibition (Fig. 6C). Collectively, miR-210 inhibition affected cytoprotection of SWT in hypoxia-induced injury.

SWT protects HL-1 cells from hypoxia-induced apoptosis by regulating $m R N A$ levels of Casp8ap2 and AIFM3. Previous studies have reported that miR-210 exerts its cardioprotective action against hypoxia-induced apoptosis by regulating Casp8ap2 and AIFM3 $(9,10)$. In the present study, RT-qPCR analysis demonstrated that miR-210 overexpression decreased the mRNA expression levels of AIFM3 and Casp8ap2, and miR-210 inhibitors increased the mRNA expression levels of AIFM3 and Casp8ap2 (Fig. S2). In order to further identify the 

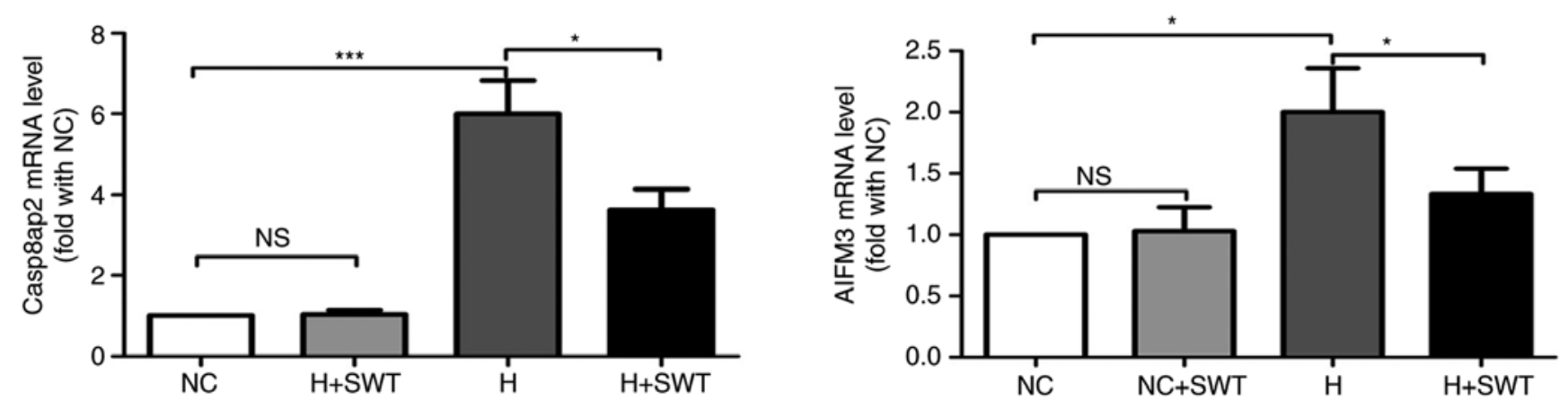

Figure 7. SWT led to a significant decrease in Casp8ap2 and AIFM3 mRNAs levels, as assessed by reverse transcription-quantitative PCR, in HL-1 cardiomyocytes exposed to hypoxia $(\mathrm{n}=3)$. Data were normalized to GAPDH and expressed relative to the normal control. ${ }^{*} \mathrm{P}<0.05,{ }^{* * * *} \mathrm{P}<0.001$. SWT, shock wave therapy; ns, not significant; NC, negative control; H, hypoxia; AIFM3, apoptosis-inducing factor mitochondrion-associated 3; Casp8ap2, caspase 8 associated protein 2.

underlying molecular mechanisms responsible for the cytoprotective effects of SWT on hypoxia-induced apoptosis in HL-1 cells, RT-qPCR was performed to detect the mRNA levels of Casp8ap2 and AIFM3. Fig. 7 demonstrates that SWT led to a significant decrease in Casp8ap2 and AIFM3 mRNA levels, which were upregulated by hypoxia.

\section{Discussion}

Using a well-established murine cardiac hypoxia model ex vivo, the present study reported that miR-210 was significantly increased following hypoxia exposure. Hypoxia-triggered myocardial injury could be alleviated by overexpression of miR-210. SWT may protect cardiomyocytes from hypoxia-induced injury by upregulating miR-210.

Ischemic injury to the myocardium results from events leading to a decrease in or interruption to coronary blood flow, including IHD. Myocardial ischemia leads to a typical pattern of metabolic and ultrastructural alterations that induce irreversible injury, causing different forms of cardiomyocyte death, referred to as apoptosis, necrosis and necroptosis $(26,27)$. Apoptosis, known as type I programmed cell death, is a highly regulated and energy-dependent process that primarily contributes to cell death in AMI, and could be initiated via the death receptor and mitochondrial pathways $(28,29)$. The mitochondrial pathway is activated by a wide range of non-receptor mediated stimuli, including hypoxia and oxidative stress (30). Apoptosis presents with a decrease in $\mathrm{Bcl}-2$ protein levels and an increase in Bax protein levels (28). Consistent with previous studies, the data from the present study demonstrated that hypoxia induced myocardial injury and apoptosis, as evidenced by decreased cell viability, increased ROS generation, decreased $\mathrm{Bcl}-2 / \mathrm{Bax}$ ratio and increased apoptotic rates.

Cardiac SWT is a novel effective therapy for IHD. Both in vivo and in vitro studies demonstrated that SWT could protect cardiomyocytes from hypoxia-induced apoptosis $(17,18)$. Similarly, the results from the present study demonstrated that SWT exhibited antiapoptotic activities. Upon hypoxia exposure, ROS originates from mitochondria and activates proapoptotic p38MAPK thereafter (31). In the present study, SWT attenuated p38MAPK activity, which was markedly increased in hypoxic cardiomyocytes.

It is well known that numerous genes are abnormally expressed in IHD, which are responsible for cardiomyocyte survival and cardiac remodeling following acute and chronic ischemia $(32,33)$. Previous studies have demonstrated that miR-210, as one of several hypoxia-induced miRNAs, plays a critical role in cell survival and possesses antiapoptotic properties (34-37). Supporting evidence suggested that miR-210 expression was ubiquitously and robustly increased in hypoxic cells (34), which is in line with the results of the present study. miR-210 overexpression decreased hypoxia-induced injury, and miR-210 inhibition aggravated hypoxic injury as evidenced by decreased cell viability and increased apoptosis. Mutharasan et al (10) demonstrated that miR-210 counteracted oxidative stress by decreasing mitochondrial ROS production and mitochondrial biogenesis in cardiomyocytes exposed to hypoxia. However, contradictory results have been reported that suggest increased expression of miR-210 in hypoxia stress activates ROS production, suppresses mitochondrial function and elevates glycolysis, leading to increased ATP levels (38). These diverging results may be attributed to cell-specific effects and varied oxygen concentrations. The data from the present study demonstrated that miR-210 overexpression decreased lipid peroxidation, which could be reversed by miR-210 inhibition. Therefore, both loss- and gain-of function experiments revealed that miR-210 was a positive regulator of cytoprotective action against hypoxia-induced injury. SWT significantly increased miR-210 expression following hypoxic insult and exerted cytoprotection, which could be prevented by miR-210 supression. To the best of our knowledge, the present study revealed for the first time that SWT prevented hypoxic injury in cardiomyocytes through regulating miRNA expression.

Both Casp8ap2 and AIFM3 have been confirmed as miR-210 targets in the heart, indicating the role of miR-210 in cell apoptosis $(10,11,39)$. Casp8ap2 is a component of the death-signaling complex that is involved in Fas-mediated apoptosis (40), and AIFM3 plays an important role in initiating the caspase-independent pathway of apoptosis (41). The present study demonstrated that SWT negatively regulated Casp8ap2 and AIFM3 in cardiomyocytes exposed to hypoxia with an increase in miR-210 expression, which may contribute to its anti-apoptotic effect.

In summary, the present study demonstrated that cardiac SWT could protect cardiomyocytes from hypoxia-induced apoptosis and oxidative stress by modulating miR-210. SWT may act as an effective therapeutic strategy for the prevention of hypoxia-induced injury in patients with IHD. 


\section{Acknowledgements}

Not applicable.

\section{Funding}

The present study was supported by grants from The Capital Health Project (grant no. Z131100004013032) and The Beijing Hospital Clinical Research 121 Project (grant no. 121-2016004).

\section{Availability of data and material}

The datasets used and/or analyzed during the current study are available from the corresponding author on reasonable request.

\section{Authors' contributions}

$\mathrm{QH}$ and TS conceived and designed the experiments; QQ, XXY and QW performed the experiments; NJ, QQ and TS analyzed the data and wrote the paper.

\section{Ethics approval and consent to participate}

Not applicable.

\section{Patient consent for publication}

Not applicable.

\section{Competing interests}

The authors declare that they have no competing interests.

\section{References}

1. Ibanez B, Heusch G, Ovize M and Van de Werf F: Evolving therapies for myocardial ischemia/reperfusion injury. $\mathrm{J}$ Am Coll Cardiol 65: 1454-1471, 2015.

2. Shepard D, VanderZanden A, Moran A, Naghavi M, Murray C and Roth G: Ischemic heart disease worldwide, 1990 to 2013 : Estimates from the global burden of disease study 2013 Circulation. Circ Cardiovasc Qual Outcomes 8: 455-456, 2015.

3. Ha M and Kim VN: Regulation of microRNA biogenesis. Nat Rev Mol Cell Biol 15: 509, 2014

4. Tang Y, Zheng J, Sun Y, Wu Z, Liu Z and Huang G: MicroRNA-1 regulates cardiomyocyte apoptosis by targeting Bcl-2. Int Heart J 50: 377-387, 2009.

5. Sayed D, He M, Hong C, Gao S, Rane S, Yang Z and Abdellatif M MicroRNA-21 is a downstream effector of AKT that mediates its antiapoptotic effects via suppression of Fas ligand. J Biol Chem 285: 20281-20290, 2010.

6. Ren XP, Wu J, Wang X, Sartor MA, Jones K, Qian J, Nicolaou P, Pritchard TJ and Fan GC: MicroRNA-320 is involved in the regulation of cardiac ischemia/reperfusion injury by targeting heat-shock protein 20. Circulation 119: 2357-2366, 2009.

7. Wang JX, Zhang XJ, Li Q, Wang K, Wang Y, Jiao JQ, Feng C, Teng S, Zhou LY, Gong Y, et al: MicroRNA-103/107 regulate programmed necrosis and myocardial ischemia/reperfusion injury through targeting FADD. Circ Res 117: 352-363, 2015.

8. Kulshreshtha R, Ferracin M, Wojcik SE, Garzon R, Alder H, Agosto-Perez FJ, Davuluri R, Liu CG, Croce CM, Negrini M, et al: A microRNA signature of hypoxia. Mol Cell Biol 27: 1859-1867, 2007.

9. Kim HW, Haider HK, Jiang S and Ashraf M: Ischemic preconditioning augments survival of stem cells via miR-210 expression by targeting caspase-8-associated protein 2 . J Biol Chem 284: 33161-33168, 2009.
10. Mutharasan RK, Nagpal V, Ichikawa $Y$ and Ardehali H: microRNA-210 is upregulated in hypoxic cardiomyocytes through Akt- and p53-dependent pathways and exerts cytoprotective effects. Am J Physiol Heart Circ Physiol 301: H1519-H1530, 2011.

11. Kim HW, Jiang S, Ashraf M and Haider KH: Stem cell-based delivery of Hypoxamir-210 to the infarcted heart: Implications on stem cell survival and preservation of infarcted heart function. J Mol Med (Berl) 90: 997-1010, 2012.

12. Fasanaro P, D'Alessandra Y, Di Stefano V, Melchionna R, Romani S, Pompilio G, Capogrossi MC and Martelli F: MicroRNA-210 modulates endothelial cell response to hypoxia and inhibits the receptor tyrosine kinase ligand Ephrin-A3. J Biol Chem 283: 15878-15883, 2008.

13. Nishida T, Shimokawa H, Oi K, Tatewaki H, Uwatoku T, Abe K, Matsumoto Y, Kajihara N, Eto M, Matsuda T, et al: Extracorporeal cardiac shock wave therapy markedly ameliorates ischemia-induced myocardial dysfunction in pigs in vivo. Circulation 110: 3055-3061, 2004.

14. Fukumoto Y, Ito A, Uwatoku T, Matoba T, Kishi T, Tanaka H, Takeshita A, Sunagawa K and Shimokawa H: Extracorporeal cardiac shock wave therapy ameliorates myocardial ischemia in patients with severe coronary artery disease. Coron Artery Dis 17: 63-70, 2006.

15. Cassar A, Prasad M, Rodriguez-Porcel M, Reeder GS, Karia D, DeMaria AN and Lerman A: Safety and efficacy of extracorporeal shock wave myocardial revascularization therapy for refractory angina pectoris. Mayo Clin Proc 89: 346-354, 2014.

16. Khattab AA, Brodersen B, Schuermann-Kuchenbrandt D, Beurich H, Tölg R, Geist V, Schäfer T and Richardt G: Extracorporeal cardiac shock wave therapy: First experience in the everyday practice for treatment of chronic refractory angina pectoris. Int J Cardiol 121: 84-85, 2007.

17. Yu W, Shen T, Liu B, Wang S, Li J, Dai D, Cai J and He Q: Cardiac shock wave therapy attenuates $\mathrm{H} 9 \mathrm{c} 2$ myoblast apoptosis by activating the AKT signal pathway. Cell Physiol Biochem 33: 1293-1303, 2014.

18. Zhang Y, Shen T, Liu B, Dai D, Cai J, Zhao C, Du L, Jia N and He Q: Cardiac shock wave therapy attenuates cardiomyocyte apoptosis after acute myocardial infarction in rats. Cell Physiol Biochem 49: 1734-1746, 2018.

19. Hamacher-Brady A, Brady NR and Gottlieb RA: Enhancing macroautophagy protects against ischemia/reperfusion injury in cardiac myocytes. J Biol Chem 281: 29776-29787, 2006.

20. Du L, Shen T, Liu B, Zhang Y, Zhao C, Jia N, Wang Q and He Q: Shock wave therapy promotes cardiomyocyte autophagy and survival during hypoxia. Cell Physiol Biochem 42: 673-684, 2017.

21. Livak KJ and Schmittgen TD: Analysis of relative gene expression data using real-time quantitative PCR and the 2(-Delta Delta C(T)) Method. 25: 402-408, 2001.

22. White SM, Constantin PE and Claycomb WC: Cardiac physiology at the cellular level: Use of cultured HL-1 cardiomyocytes for studies of cardiac muscle cell structure and function. Am J Physiol Heart Circ Physiol 286: H823-H829, 2004.

23. Levraut J, Iwase H, Shao ZH, Hoek TLV and Schumacker PT: Cell death during ischemia: Relationship to mitochondrial depolarization and ROS generation. Am J Physiol Heart Circ Physiol 284: H549-H558, 2003.

24. Pryor WA and Stanley JP: Suggested mechanism for the production of malonaldehyde during the autoxidation of polyunsaturated fatty acids. Nonenzymic production of prostaglandin endoperoxides during autoxidation. J Org Chem 40: 3615-3617, 1975.

25. Chan SY and Loscalzo J: MicroRNA-210: A unique and pleiotropic hypoxamir. Cell Cycle 9: 1072-1083, 2010.

26. Kang Peter M, Haunstetter A, Aoki H, Usheva A and Izumo S: Morphological and molecular characterization of adult cardiomyocyte apoptosis during hypoxia and reoxygenation. Circ Res 87: 118-125, 2000.

27. Buja LM: Myocardial ischemia and reperfusion injury. Cardiovasc Pathol 14: 170-175, 2005.

28. Misao J, Hayakawa Y, Ohno M, Kato S, Fujiwara T and Fujiwara H: Expression of bcl-2 protein, an inhibitor of apoptosis, and Bax, an accelerator of apoptosis, in ventricular myocytes of human hearts with myocardial infarction. Circulation 94: 1506-1512, 1996.

29. Olivetti G, Quaini F, Sala R, Lagrasta C, Corradi D, Bonacina E, Gambert SR, Cigola E and Anversa P: Acute myocardial infarction in humans is associated with activation of programmed myocyte cell death in the surviving portion of the heart. $\mathrm{J} \mathrm{Mol}$ Cell Cardiol 28: 2005-2016, 1996. 
30. Yang BC, Zander DS and Mehta JL: Hypoxia-reoxygenationinduced apoptosis in cultured adult rat myocytes and the protective effect of platelets and transforming growth factor-beta(1). J Pharmacol Exp Ther 291: 733-738, 1999.

31. See F, Thomas W, Way K, Tzanidis A, Kompa A, Lewis D, Itescu $\mathrm{S}$ and Krum H: p38 mitogen-activated protein kinase inhibition improves cardiac function and attenuates left ventricular remodeling following myocardial infarction in the rat. J Am Coll Cardiol 44: 1679-1689, 2004.

32. Lima J, Batty JA, Sinclair H and Kunadian V: MicroRNAs in ischemic heart disease: From pathophysiology to potential clinical applications. Cardiol Rev 25: 117-125, 2017.

33. Boon RA and Dimmeler S: MicroRNAs in myocardial infarction. Nat Rev Cardiol 12: 135-142, 2015.

34. Hu S, Huang M, Li Z, Jia F, Ghosh Z, Lijkwan MA, Fasanaro P, Sun N, Wang X, Martelli F, et al: MicroRNA-210 as a novel therapy for treatment of ischemic heart disease. Circulation 122 (11 Suppl): S124-S131, 2010.

35. Arif M, Pandey R, Alam P, Jiang S, Sadayappan S, Paul A and Ahmed RPH: MicroRNA-210-mediated proliferation, survival, and angiogenesis promote cardiac repair post myocardial infarction in rodents. $\mathrm{J}$ Mol Med (Berl) 95: 1369-1385, 2017.

36. Li T, Song X, Zhang J, Zhao L, Shi Y, Li Z, Liu J, Liu N, Yan Y, Xiao Y, et al: Protection of human umbilical vein endothelial cells against oxidative stress by MicroRNA-210. Oxid Med Cell Longev 2017: 3565613, 2017.
37. Diao H, Liu B, Shi Y, Song C, Guo Z, Liu N, Song X, Lu Y, Lin X and Li Z: MicroRNA-210 alleviates oxidative stress-associated cardiomyocyte apoptosis by regulating BNIP3. Biosci Biotechnol Biochem 81: 1712-1720, 2017.

38. Chen Z, Li Y, Zhang H, Huang P and Luthra R: Hypoxia-regulated microRNA-210 modulates mitochondrial function and decreases ISCU and COX10 expression. Oncogene 29: 4362-4368, 2010.

39. Kim HW, Mallick F, Durrani S, Ashraf M, Jiang S and Haider KH: Concomitant activation of miR-107/PDCD10 and hypoxamir-210/Casp8ap2 and their role in cytoprotection during ischemic preconditioning of stem cells. Antioxid Redox Signal 17: 1053-1065, 2012.

40. Imai Y, Kimura T, Murakami A, Yajima N, Sakamaki K and Yonehara S: The CED-4-homologous protein FLASH is involved in Fas-mediated activation of caspase-8 during apoptosis. Nature 398: 777-785, 1999.

41. Xie Q, Lin T, Zhang Y, Zheng J and Bonanno JA: Molecular cloning and characterization of a human AIF-like gene with ability to induce apoptosis. J Biol Chem 280: 19673-19681, 2005.

(i) $(-)$ This work is licensed under a Creative Commons Attribution-NonCommercial-NoDerivatives 4.0 International (CC BY-NC-ND 4.0) License. 